\title{
Effect of Volume Ratio of Heat Rejection Process on Performance of an Atkinson Cycle
}

\author{
R. EBRAHIMI* \\ Department of Mechanical Engineering of Biosystem, Shahrekord University, P.O. Box 115, Shahrekord, Iran \\ (Received April 26, 2016; in final form November 19, 2017)

\begin{abstract}
The objective of this study is to analyze the effect of volume ratio of heat rejection process on the performance
\end{abstract} \\ of dual cycle. Using finite-time thermodynamics, the relations between the volume ratio of heat rejection, the \\ thermal efficiency, the power output, the heat transfer losses, the friction power and the compression ratio for an \\ air standard Atkinson cycle have been derived. In the model, the nonlinear relation between the specific heats \\ of working fluid and its temperature, the frictional loss and heat leakage loss are considered. The results show \\ that the power output and the thermal efficiency first increase with the increase of volume ratio of heat rejection \\ process and then start to decrease. The optimum value of the volume ratio of heat rejection which maximizes \\ the power output is higher than that which maximizes the thermal efficiency, while the optimum value of the \\ compression ratio which maximizes the power output is lower than that which maximizes the thermal efficiency. \\ The results obtained in the present study provide guidance to the performance evaluation and improvement for \\ practical internal combustion engines.
}

DOI: 10.12693/APhysPolA.133.201

PACS/topics: Atkinson cycle, heat rejection process

\section{Introduction}

A series of achievements have been made since finitetime thermodynamics was used to analyze and optimize the performance of real thermodynamic processes, devices and cycles [1-5]. The Atkinson cycle, one of the most heat-efficient, high-expansion ratio cycles, is designed by James Atkinson in 1882 [6] to provide efficiency at the expense of power. Chen and Lin [7] maximized the power density of an Atkinson engine with respect to the maximum cycle-temperature ratio. AlSarkhi and Akash [8] compared the performance characteristic curves of the Atkinson cycle with those of the Miller and Joule-Brayton cycles by using numerical examples, and outlined the effect of maximizing power density on the performance of the cycle efficiency. Wang and Hou [9] studied the performance analysis and comparison of an Atkinson cycle coupled to variable temperature heat reservoirs under maximum power and maximum power density conditions, assuming a constant specific heat, too. Chen et al. [10] built a class of generalized irreversible universal steady flow heat engine cycle model consisting of two heating branches, two cooling branches, and two adiabatic branches with consideration of the losses of heat resistance, heat leakage, and internal irreversibility. The performance characteristics of Diesel, Otto, Brayton, Atkinson, dual and Miller cycles were derived. Hou [11] compared the performances of the airstandard Otto and Atkinson cycles by taking into consideration the heat transfer impacts. Ebrahimi $[12,13]$ advanced specific heat ratio varied with temperature with

\footnotetext{
*e-mail: Rahim.Ebrahimi@gmail.com
}

nonlinear relation model, studied the power output and efficiency performance characteristics of an irreversible Atkinson cycle, and investigated the influence of piston mean velocity, cylinder wall temperature, stroke length and volume efficiency on cycle performance. The performance characteristics of six endoreversible heat engines, including Carnot, Diesel, Otto, Atkinson, Brayton and Dual cycles is studied by Ding et al. [14]. Gahruei et al. [15] carried out a comparative performance analysis for classical dual and dual-Atkinson cycles based on finite-time thermodynamics, taking into account variable specific heats of the working fluid and the losses resulting from heat transfer and frictions. Ebrahimi [16] derived the power output and the thermal efficiency performance characteristics of an irreversible Atkinson cycle, investigated the influence of air-fuel ratio, fuel mass flow rate and residual gas on cycle performance, and found that the performances would increase with increase in air-fuel ratio and residual gas when the compression ratio was less than certain value, the performances would decrease with increase in air-fuel ratio and residual gas when the compression ratio exceeded certain value, and the performance would increase with increase in fuel mass flow rate throughout the compression ratio working range. The influences of the design and running parameters on the performance characteristics of an Atkinson engine are investigated by Gonca [17]. A comprehensive parametrical study is conducted. The results showed that the effective power, effective power density, and effective efficiency rise with rising air inlet pressure, cycle pressure and temperature ratios.

As can be seen in the relevant literature, the investigations of the effect of volume ratio of heat rejection process on performance of Atkinson cycle do not appear to have been published. Therefore, the objective of this study is 
to examine the effect of volume ratio of heat rejection process on the performance of air standard Atkinson cycle.

\section{An irreversible Atkinson cycle model}

The pressure-volume $P-V$ and the temperatureentropy $T-S$ diagrams of an irreversible Atkinson heat engine is shown in Fig. 1 , where $T_{1}, T_{2}, T_{3}, T_{4}$ and $T_{5}$ are the temperatures of the working substance in state points 1, 2, 3 and 4, 5. Process $1 \rightarrow 2$ is an isentropic compression. The heat addition occurs in the constant volume process $2 \rightarrow 3$. The process $3 \rightarrow 4$ is an isentropic expansion process. The heat-removing process is the reversible constant pressure $4 \rightarrow 1$.

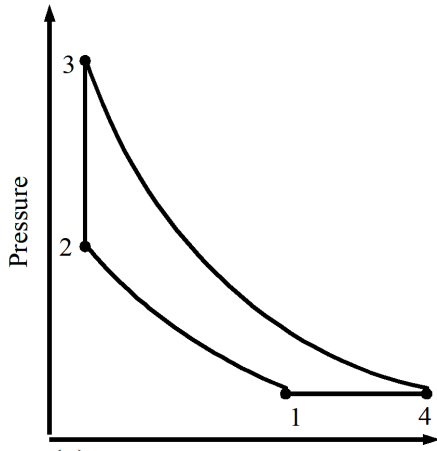

(a)

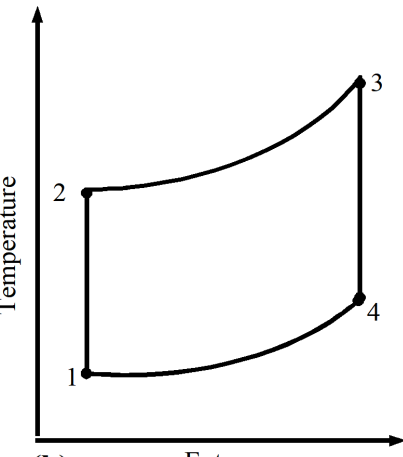

(b)

Entropy
Fig. 1. (a) $P-V$ diagram, (b) $T-S$ diagram for the air standard Atkinson cycle.

In most cycle models, the working fluid is assumed to behave as an ideal gas with constant specific heats. But this assumption can be valid only for small temperature difference. For the large temperature difference encountered in the practical cycle, this assumption cannot be applied. According to Refs. [3] and [18], for the temperature range of 300-3500 K, the specific heat with constant volume can be written as

$$
\begin{aligned}
& C_{v}=2.506 \times 10^{-11} T^{2}+1.454 \times 10^{-7} T^{1.5} \\
& -4.245 \times 10^{-7} T+3.162 \times 10^{-5} T^{0.5}+1.0433 \\
& -1.512 \times 10^{4} T^{-1.5}+3.063 \times 10^{5} T^{-2} \\
& \quad-2.212 \times 10^{-7} T^{-3},
\end{aligned}
$$

where $T$ is the absolute temperature is in $[\mathrm{K}]$ and $C_{v}$ is expressed in $[\mathrm{J} / \mathrm{kg} \mathrm{K}]$.

According to the relation between specific heat with constant pressure and specific heat with constant volume, the specific heat with constant volume can be written as

$$
C_{p}=C_{v}+R_{\text {air }}=C_{v}+0.287,
$$

where $R_{\text {air }}=0.287 \mathrm{~kJ} / \mathrm{kg}$ is the gas constant of the working fluid.

The heat added per second to the working fluid during process $2 \rightarrow 3$ is

$$
Q_{i n}=\dot{m}_{t} \int_{T_{2}}^{T_{3}} C_{v} \mathrm{~d} T=\dot{m}_{t}\left[8.353 \times 10^{-12} T^{3}\right.
$$

$$
\begin{aligned}
& +5.818 \times 10^{-8} T^{2.5}-2.123 \times 10^{-7} T^{2} \\
& +2.108 \times 10^{-5} T^{1.5}+1.0433 T+3.024 \times 10^{4} T^{-0.5} \\
& \left.-3.063 \times 10^{5} T^{-1}+1.106 \times 10^{-7} T^{-2}\right]_{T_{2}}^{T_{3}}
\end{aligned}
$$

The heat rejected per second by the working fluid during processes $4 \rightarrow 5$ and $5 \rightarrow 1$ is

$$
\begin{aligned}
& Q_{\text {out }}=\dot{m}_{t} \int_{T_{1}}^{T_{4}} C_{p} \mathrm{~d} T=\dot{m}_{t}\left[8.353 \times 10^{-12} T^{3}\right. \\
& \quad+5.818 \times 10^{-8} T^{2.5}-2.123 \times 10^{-7} T^{2} \\
& \quad+2.108 \times 10^{-5} T^{1.5}+1.3303 T+3.024 \times 10^{4} T^{-0.5} \\
& \left.\quad-3.063 \times 10^{5} T^{-1}-1.106 \times 10^{-7} T^{-2}\right]_{T_{1}}^{T_{4}},
\end{aligned}
$$

where $\dot{m}_{t}$ is the mass flow rate of the working fluid and the unit of $\dot{m}_{t}$ is $[\mathrm{kg} / \mathrm{s}]$.

Since $C_{p}$ and $C_{v}$ are dependent on temperature, adiabatic exponent $\gamma=C_{p} / C_{v}$ will vary with temperature. According to Refs. [12] and [19] for small change in temperature $\mathrm{d} T$, and volume $\mathrm{d} V$ of the working fluid, the equation for reversible adiabatic process with variable $\gamma$ can be written as follows:

$$
(T+\mathrm{d} T)(V+\mathrm{d} V)^{\gamma-1}=T V^{\gamma-1}
$$

expanding the right-hand term, one arrives to an alternative form as

$$
\begin{aligned}
& (T+\mathrm{d} T)(V+\mathrm{d} V)^{\gamma-1}=(T+\mathrm{d} T)\left(V^{\gamma-1}\right. \\
& \left.+(\gamma-1) V^{\gamma-2} \mathrm{~d} V+\ldots \underset{\approx 0}{\mathrm{~d} V^{2}}+\ldots+\ldots \mathrm{d}_{\approx 0}^{\gamma-1}\right) \mathrm{d} V= \\
& \quad(T+\mathrm{d} T)\left(V^{\gamma-1}+(\gamma-1) V^{\gamma-2} \mathrm{~d} V\right)= \\
& T V^{\gamma-1}+(\gamma-1) T V^{\gamma-2} \mathrm{~d} V+V^{\gamma-1} \mathrm{~d} T \\
& +\underset{\approx 0}{(\gamma-1) \mathrm{d} V^{\gamma-2} \mathrm{~d} V \mathrm{~d} T}=T V^{\gamma-1} \\
& +(\gamma-1) T V^{\gamma-2} \mathrm{~d} V+V^{\gamma-1} \mathrm{~d} T .
\end{aligned}
$$

By substituting Eq. (6) into Eq. (5), we finally get

$$
C_{v} \frac{\mathrm{d} T}{T}=-R_{\text {air }} \frac{\mathrm{d} V}{V} .
$$

Integrating Eq. (7) in process $\mathrm{i} \rightarrow \mathrm{j}$, we have

$$
C_{v} \ln \frac{T_{j}}{T_{i}}=R_{\text {air }} \ln \frac{V_{i}}{V_{j}},
$$

where the temperature in the equation of $C_{v}$ is defined as:

$$
T=\left(T_{j}-T_{i}\right) / \ln \left(T_{j} / T_{i}\right) .
$$

The volume ratio of heat rejection, $\psi$, the effective compression ratio, $r_{c}^{*}$, and the compression ratio, $r_{c}$, are defined as:

$$
\begin{aligned}
& \psi=\frac{V_{4}}{V_{1}}=\frac{T_{4}}{T_{1}}, \\
& r_{c}^{*}=\frac{V_{1}}{V_{2}}
\end{aligned}
$$

and

$$
r_{c}=\frac{V_{4}}{V_{2}}=\psi r_{c}^{*} .
$$

Therefore, equations for reversible adiabatic processes 
$1 \rightarrow 2$ and $3 \rightarrow 4$ are as follows:

$$
\begin{aligned}
& C_{v} \ln \frac{T_{2}}{T_{1}}=R_{\text {air }} \ln r_{c}^{*}, \\
& C_{v} \ln \frac{T_{3}}{T_{4}}=R_{\text {air }} \ln \left(\psi r_{c}^{*}\right) .
\end{aligned}
$$

For an ideal reciprocating heat engine cycle model, there are no losses. However, for a real reciprocating heat engine cycle, heat transfer irreversibility between the working fluid and the cylinder wall $\left(\dot{Q}_{h t}\right)$ is not negligible. It can be assumed that the heat loss through the cylinder wall is proportional to the average temperature of both the working fluid and the cylinder wall and that the wall temperature is constant at $T_{0}$ [12]. If the released heat by combustion for one mass working fluid is $A$ and the heat leakage coefficient of the cylinder wall is $B$, the heat added to the working fluid by combustion is given by the linear relation

$$
\dot{Q}_{i n}=\dot{m}_{t} A-\dot{Q}_{h t}=\dot{m}_{t}\left[A-B\left(T_{2}+T_{4}-2 T_{0}\right)\right] .
$$

Every time the piston moves, friction acts to retard the motion. Considering the friction effects on the piston in all the processes of the cycle, we assume a dissipation term represented by a friction force that is linearly proportional to the velocity of the piston, which can be written as follows $[5,20]$ :

$$
f_{f}=f \nu=f \frac{\mathrm{d} x}{\mathrm{~d} t}
$$

where $f$ is a coefficient of friction that takes into account the global losses and $x$ is the piston displacement. Therefore, the friction power is

$$
P_{f}=\frac{\mathrm{d} W_{f}}{\mathrm{~d} t}=f \frac{\mathrm{d} x}{\mathrm{~d} t} \frac{\mathrm{d} x}{\mathrm{~d} t}=f \nu^{2} .
$$

The piston mean velocity is

$$
\bar{\nu}=\frac{x_{1}-x_{2}}{\Delta t_{12}}=\frac{x_{2}\left(r_{c}-1\right)}{\Delta t_{12}},
$$

where $x_{2}$ is the piston position at minimum volume and $\Delta t_{12}$ is the time spent in the power stroke. Thus, the friction power is

$$
P_{f}=b\left(r_{c}-1\right)^{2} .
$$

The power output of the cycle is

$$
\begin{aligned}
& P_{\text {out }}=\dot{Q}_{\text {in }}-\dot{Q}_{\text {out }}-P_{f}=\dot{m}_{t}\left[8.353 \times 10^{-12}\left(T_{1}^{3}+T_{3}^{3}\right.\right. \\
& \left.\quad-T_{2}^{3}-T_{4}^{3}\right)+5.818 \times 10^{-8}\left(T_{1}^{2.5}+T_{3}^{2.5}-T_{2}^{2.5}\right. \\
& \left.\quad-T_{4}^{2.5}\right)-2.123 \times 10^{-7}\left(T_{1}^{2}+T_{3}^{2}-T_{2}^{2}-T_{4}^{2}\right) \\
& \quad+2.108 \times 10^{-5}\left(T_{1}^{1.5}+T_{3}^{1.5}-T_{2}^{1.5}-T_{4}^{1.5}\right) \\
& \quad+1.0433\left(T_{3}-T_{2}\right)+1.3303\left(T_{1}-T_{4}\right) \\
& \quad+3.024 \times 10^{4}\left(T_{1}^{-0.5}+T_{3}^{-0.5}-T_{2}^{-0.5}-T_{4}^{-0.5}\right) \\
& \quad-3.063 \times 10^{5}\left(T_{1}^{-1}+T_{3}^{-1}-T_{2}^{-1}-T_{4}^{-1}\right) \\
& \left.\quad+1.106 \times 10^{7}\left(T_{1}^{-2}+T_{3}^{-2}-T_{2}^{-2}-T_{4}^{-2}\right)\right] \\
& \quad-b\left(r_{c}-1\right)^{2} . \\
& \dot{Q}_{i n}=\dot{m}_{t}\left[8.353 \times 10^{-12}\left(T_{3}^{3}-T_{2}^{3}\right)+5.818\right.
\end{aligned}
$$

$$
\begin{aligned}
& \times 10^{-8}\left(T_{3}^{2.5}-T_{2}^{2.5}\right)-2.123 \times 10^{-7}\left(T_{3}^{2}-T_{2}^{2}\right) \\
& +2.108 \times 10^{-5}\left(T_{3}^{1.5}-T_{2}^{1.5}\right)+1.0433\left(T_{3}-T_{2}\right) \\
& +3.024 \times 10^{4}\left(T_{3}^{-0.5}-T_{2}^{-0.5}\right)-3.063 \times 10^{5}\left(T_{3}^{-1}\right. \\
& \left.\left.-T_{2}^{-1}\right)+1.106 \times 10^{7}\left(T_{3}^{-2}-T_{2}^{-2}\right)\right] .
\end{aligned}
$$

The thermal efficiency of the cycle is

$$
\eta_{t h}=\frac{P_{\text {out }}}{\dot{Q}_{\text {in }}} .
$$

When $r_{c}^{*}, T_{0}, R_{a i r}, T_{1}, A, B$ and $\dot{m}_{t}$ are given, $T_{2}$ can be obtained from Eq. (13); then, substituting from Eq. (3) into Eq. (15) yields $T_{3}$; and $T_{4}$ can be found from Eq. (14). Substituting $T_{1}, T_{2}, T_{3}$ and $T_{4}$ into Eqs. (20) and (21) yields the power output and thermal efficiency. Therefore, the relations between the power output, the thermal efficiency and the engine parameters can be derived.

\section{Results and discussion}

The following constants and parameter values have been used in this exercise: $T_{1}=300 \mathrm{~K}, T_{0}=400 \mathrm{~K}$, $R_{\text {air }}=0.287 \mathrm{~kJ} /(\mathrm{kg} \mathrm{K}), B=0.4 \mathrm{~kJ} /(\mathrm{kg} \mathrm{K}), b=$ $0.012 \mathrm{~kW}, A=2820.5 \mathrm{~kJ} / \mathrm{kg}, r_{c}^{*}=1.1 \rightarrow 15$, and $\dot{m}_{t}=0.0624 \mathrm{~kg} / \mathrm{s}[3-5,17-22]$. Using the above constants and range of parameters, the power output versus compression ratio characteristic, the power output versus volume ratio of heat rejection characteristic, the thermal efficiency versus compression ratio characteristic, the thermal efficiency versus volume ratio of heat rejection characteristic, the friction power versus compression ratio characteristic, the friction power versus volume ratio of heat rejection characteristic, the heat leakage loss versus compression ratio characteristic, the heat leakage loss versus volume ratio of heat rejection characteristic, the thermal efficiency versus power output characteristic, the thermal efficiency versus friction power characteristic, the ratio of the heat added by the working fluid to the heat rejected by the working fluid versus power output characteristic and the ratio of the heat added by the working fluid to the heat rejected by the working fluid versus friction power characteristic with varying the effective compression ratio can be plotted as in Figs. 2-4. One can see that the power output versus compression ratio characteristic and the efficiency versus compression ratio characteristic are parabolic like curves, and the power output versus efficiency curve is loop shaped. From these figures, it can be also found that the volume ratio of heat rejection process plays important roles on the performance cycle. They reflect the performance characteristics of a real irreversible Atkinson cycle. Numerical examples are shown as follows.

In Fig. 2, changes of the volume ratio of heat rejection process, compression ratio, power output and the thermal efficiency can be seen. One can see that the power output and the efficiency reach their maximum (the peak of each curve) while the volume ratio of heat rejection and the 


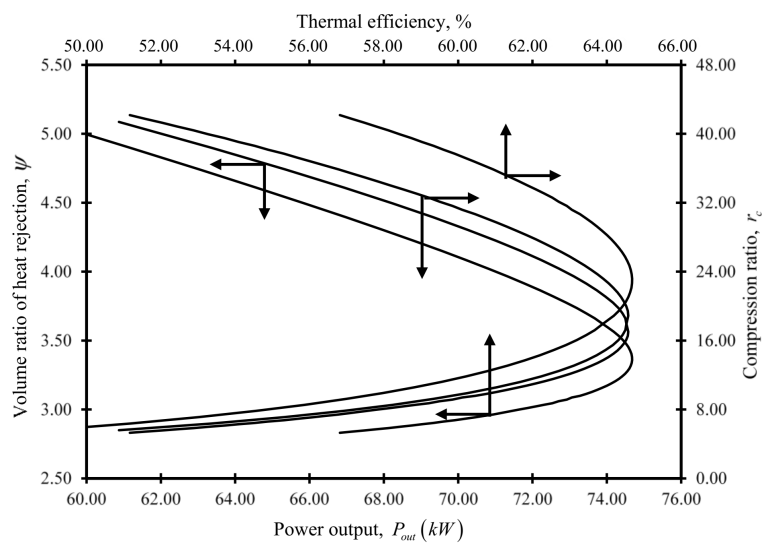

Fig. 2. The variations of $P_{\text {out }}-\psi-r_{c}$ and $\eta_{t h}-\psi-r_{c}$.

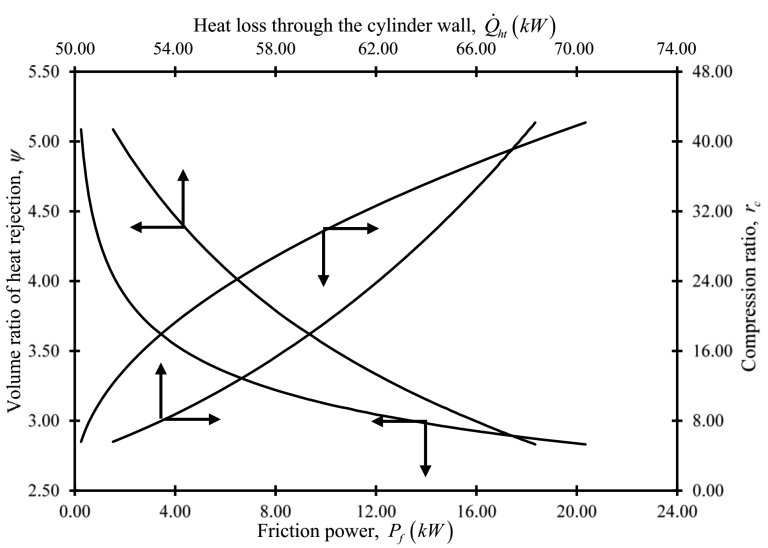

Fig. 3. The variations of $P_{f}-\psi-r_{c}$ and $\dot{Q}_{h t}-\psi-r_{c}$.

compression ratio reach optimum values. It is clear from the figure that the optimum value of the volume ratio of heat rejection which maximizes the power output is higher than that which maximizes the thermal efficiency, while the optimum value of the compression ratio which maximizes the power output is lower than that which maximizes the thermal efficiency.

The variations of the volume ratio of heat rejection process and compression ratio with respect to the friction power and the heat leakage loss are shown in Fig. 3. The figure show that the heat leakage loss and the friction power decrease with respect to increasing volume ratio of heat rejection, while the heat leakage loss and the friction power increase with increasing compression ratio.

The variation of the thermal efficiency and ratio of the heat added by the working fluid to the heat rejected by the working fluid with respect to the power output and friction power are shown in Fig. 4. It is clear that there are two values of power output and friction power at each thermal efficiency and also there are two values of ratio of the heat added by the working fluid to the heat rejected by the working fluid at each power output. The ratio of the heat added by the working fluid to the heat rejected

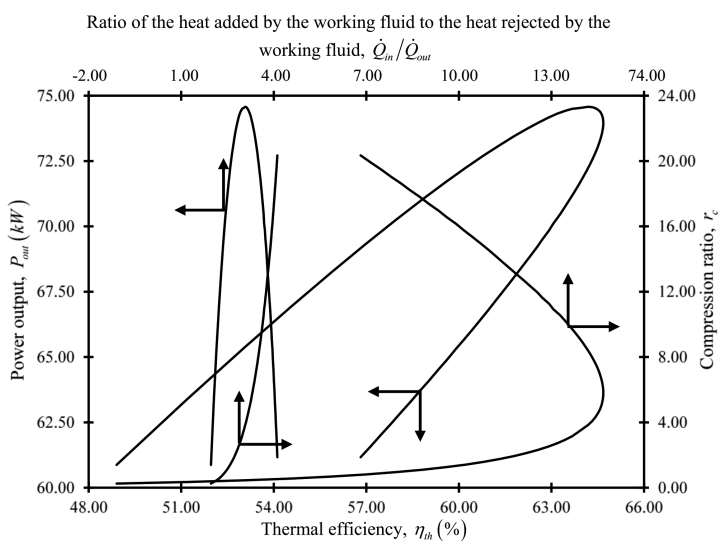

Fig. 4. The variations of $\eta_{t h}-P_{\text {out }}-P_{f}$ and $\dot{Q}_{\text {in }} / \dot{Q}_{\text {out }}-P_{\text {out }}-P_{f}$.

by the working fluid increase with increase friction power.

According to above analysis, it can be found that the effect of the volume ratio of heat rejection process on the cycle performance is obvious, and it should be considered in practice cycle analysis in order to make the cycle model be more close to practice.

\section{Conclusions}

In this study, finite-time thermodynamics analysis of an irreversible air standard Atkinson cycle is established which is closer to practice engine. In this model, effect of volume ratio of heat rejection process by considering the nonlinear relation between the specific heats of working fluid and its temperature, the frictional loss and heat transfer loss is analyzed. Numerical examples displayed the influences of this parameter in power output, thermal efficiency, friction power and heat leakage loss of the cycle.

\section{Acknowledgments}

This paper is supported by Shahrekord University through grant No. 95GRN1M1916.

\section{References}

[1] Y. Ge, L. Chen, F. Sun, C. Wu, Int. Commun. Heat Mass Transf. 32, 1045 (2005).

[2] M. Mozurkewich, R.S. Berry, J. Appl. Phys. 53, 34 (1982).

[3] R. Ebrahimi, Acta Phys. Pol. A 122, 645 (2012).

[4] R. Ebrahimi, J. Energy Inst. 84, 30 (2011).

[5] R. Ebrahim, J. Energy Inst. 84, 38 (2011).

[6] Y. Zhao, J. Chen, Appl. Energy 83, 789 (2006).

[7] L. Chen, J. Lin, Ener. Convers. Manage. 39, 337 (1998).

[8] A. Al-Sarkhi, B.A. Akash, Int. Commun. Heat Mass Transf. 29, 1159 (2002). 
[9] P. Wang, S.S. Hou, Ener. Convers. Manage. 46, 2637 (2005)

[10] L. Chen, W. Zhang, F. Sun, Appl. Energy 84, 512 (2007).

[11] S. Hou, Energy Convers. Manage. 48, 1683 (2007).

[12] R. Ebrahimi, R. Math. Comput. Model. 53, 1289 (2011).

[13] R. Ebrahimi, L. Chen, Int. J. Amb. Energy 31, 101 (2010).

[14] Z. Ding, L. Chen, F. Sun, Appl. Math. Model. 35, 728 (2011).

[15] M.H. Gahruei, H.S. Jeshvaghani, S. Vahidi, L. Chen, Appl. Math. Model. 37, 7319 (2013).
[16] R. Ebrahimi, Acta Phys. Pol. A 124, 29 (2013).

[17] G. Gonca, Acta Phys. Pol. A 132, 1306 (2017).

[18] R. Sonntag, C. Borgnakke, G. Van Wylen, Fundamentals of Thermodynamics, 5th ed., Wiley, New York 1998.

[19] R. Ebrahimi, M. Sherafati, J. Therm. Anal. Calorim. 111, 951 (2013).

[20] R. Ebrahimi, Acta Phys. Pol. A 118, 534 (2010).

[21] R. Ebrahimi, Acta Phys. Pol. A 120, 384 (2011).

[22] R. Ebrahimi, M. Mercier, IJE Trans. B Appl. 24, 65 (2011). 